\title{
Linx
}

Revue des linguistes de l'université Paris X Nanterre

$79 \mid 2019$

Enseigner et apprendre à interagir en langue étrangère : réflexions linguistiques et didactiques

\section{Nouvelles ressources pour le FLE à partir des études en interaction}

New resources for teaching French as a Foreign Language based on studies of talk-in-interaction

\section{Elisa RAVAZZOLO et Carole ETIENNE}

\section{OpenEdition}

\section{Journals}

Édition électronique

URL : http://journals.openedition.org/linx/3454

DOI : 10.4000/linx.3454

ISSN : 2118-9692

\section{Éditeur}

Presses universitaires de Paris Nanterre

\section{Référence électronique}

Elisa RAVAZZOLO et Carole ETIENNE, « Nouvelles ressources pour le FLE à partir des études en interaction », Linx [En ligne], 79 | 2019, document 2, mis en ligne le 30 décembre 2019, consulté le 07 avril 2020. URL : http://journals.openedition.org/linx/3454; DOI : https://doi.org/10.4000/linx.3454

Ce document a été généré automatiquement le 7 avril 2020.

Département de Sciences du langage, Université Paris Ouest 


\title{
Nouvelles ressources pour le FLE à partir des études en interaction
}

\author{
New resources for teaching French as a Foreign Language based on studies of \\ talk-in-interaction
}

Elisa RAVAZZOLO et Carole ETIENNE

\section{Les objectifs du projet}

1 L'équipe LIS du laboratoire ICAR ${ }^{1}$, spécialiste de l'étude des interactions, recueille, documente et transcrit depuis plus d'une quarantaine d'années des situations sociales ordinaires dans une grande variété de contextes que la banque de données CLAPI ${ }^{2}$ a rendu disponibles en libre accès à la communauté depuis 2005. Véronique Traverso, alors directrice de l'équipe LIS, a décidé de constituer il y a environ une quinzaine d'années un réseau de partenaires didacticiens et enseignants de français et de linguistique française afin de concevoir ensemble une solution pour transposer les travaux de recherche en interaction en ressources adaptées à l'enseignement du français oral.

2 Dans cet article nous présenterons l'approche méthodologique qui a conduit à la constitution de la plateforme CLAPI-FLE ${ }^{3}$ dédiée à l'apprentissage des interactions. Nous détaillerons ensuite plusieurs exploitations de ressources pédagogiques issues de CLAPI-FLE, en situation de classe, pour différents objectifs pédagogiques, et à l'attention de différents publics d'apprenants dans des enseignements de français et de linguistique française. 


\section{L'analyse des interactions et la didactique des langues}

\subsection{L'analyse des interactions}

3 Nos travaux s'inscrivent dans le champ de la linguistique de corpus où le chercheur construit ses études à partir de l'analyse des corpus oraux, donc des données de terrain, afin de déduire des récurrences dans les actions langagières et l'ensemble des ressources mises en jeu par les locuteurs pour mener à bien leurs échanges. D'un niveau de granularité assez fin, les actions langagières peuvent se décrire par des fonctions d'ordre micro que les locuteurs vont remplir (demander un produit, hésiter, douter, confirmer, payer) pour mener à bien une activité interactionnelle plus étendue (achat d'un produit). Sont considérées comme ressources des procédés aussi variés que le lexique ; l'usage de marqueurs spécifiques de l'oral employés en grand nombre qui, utilisés seuls ou cumulés, vont modifier le sens d'une production, voire prendre la valeur d'un tour de parole; la superposition de parole (chevauchement) ; la prosodie ; des structures syntaxiques comme les dislocations; le non verbal avec les bruits-sons (hum, tsk, pss, etc.) qui remplacent ou accompagnent une production lexicale; les différents gestes (déplacements, orientation du corps vers un locuteur, pointage, manipulation d'objets, etc.), regards ou mimiques ainsi que des procédés complexes comme la reformulation ou la répétition pour ne citer que les principaux.

Cette approche de l'étude de la conversation a pour fondements les travaux sur l'organisation de la parole (Sacks, Schegloff \& Jefferson 1974) et les pratiques sociales situées (Garfinkel 1984 ; Pekarek Doehler 2005). Dans l'équipe LIS qui cherche à décrire le français parlé en interaction (Traverso 2016) les thématiques suivantes ont été particulièrement étudiées : les interactions dans les commerces (Kerbrat-Orecchioni \& Traverso 2009), les conversations familières (Traverso 1998), les invitations téléphoniques (Traverso, Ticca, Ursi 2018), les interactions dans le domaine de la santé (Ticca \& Traverso 2015), les conversations en ligne (Traverso 2011), les procédés d'hétéro-répétitions (groupe Icor 2018) ou la multimodalité (Mondada 2014 ; 2017).

5 L'analyse des interactions en tant que pratique sociale située met en évidence la conception dynamique de la langue où « tout se construit » au cours de l'interaction dans une séquence temporelle « on line » et où la production et la réception s'ajustent et se modifient en permanence. Les locuteurs doivent s'adapter au contexte mais ils le modifient également en retour. Pour mener à bien leurs échanges, ils mobilisent une large variété de ressources dont la parole, les regards, les gestes, et se déplacent ou manipulent des artefacts suivant la situation dans laquelle ils sont engagés.

\subsection{Les corpus oraux d'interactions en didactique des langues}

6 L'utilisation des corpus écologiques d'interactions de CLAPI dans l'enseignement cherche à familiariser les apprenants au français tel qu'il est parlé en interaction dans les situations sociales du quotidien, en d'autres termes elle cherche à se rapprocher d'une situation d'immersion que tous les spécialistes de l'apprentissage des langues recommandent, sachant que les apprenants n'ont pas tous la possibilité de séjourner en France ou dans un pays francophone. À cet argument, certains opposent parfois le caractère complexe de ces données où le débit réel des participants et les mécanismes 
naturels de l'oral (marqueurs, tours de parole courts, hésitations, bruits ambiants, superpositions de parole, etc.) rendraient difficile la compréhension. Néanmoins, en situation réelle d'immersion, un apprenant est bien confronté à ces mécanismes qu'il doit assimiler en temps réel. Il n'a pas la possibilité de ré-écouter l'échange ou de connaitre au préalable le contexte ou la liste des mots et des expressions difficiles. C'est à ces mécanismes spécifiques de l'oral que la plateforme CLAPI-FLE cherche à sensibiliser les apprenants en proposant aux enseignants et didacticiens des ressources utilisables dans les différentes étapes de l'apprentissage dès le niveau débutant.

Dès les années 80 , les chercheurs préconisaient l'utilisation de documents authentiques pour apprendre la langue selon la théorie de l'input (Krashen \& Tracy 1983) qui établissait le lien entre " entendre et comprendre des productions de natifs " et l'acquisition de la langue. Puis, il y a eu le développement du Data Driven Learning avec les travaux de Johns (1991), d'Aston (2001) et de l'équipe du Crapel (Boulton 2009, Debaisieux 2009) où, dans le processus d'apprentissage, les connaissances s'acquièrent par l'exploration des données. Cette approche donne accès aux pratiques réelles des locuteurs, attestées dans les corpus oraux, qui peuvent ainsi être comprises et assimilées pour disposer de plusieurs réalisations au lieu de données orales construites, normalisant un format unique qui ne correspond ni à l'organisation ni au contenu réel des productions des natifs. Plus récemment, Giroud \& Surcouf (2016) ont comparé les enregistrements proposés dans différents manuels destinés aux débutants avec de l'oral spontané.

8 Au-delà de l'utilisation de données réellement attestées, le courant du data-driven learning a étudié de quelle manière l'apprenant pouvait devenir acteur de son propre apprentissage en " autonomous learning " (Aston 2001) en s'appropriant des outils de la linguistique comme le concordancier, pour comprendre un usage en contexte ou identifier des collocations et des récurrences (André 2016), mais également d'autres procédés pour répondre à une large variété d'objectifs pédagogiques en appréhendant la langue dans la diversité de ses représentations (Boulton \& Tyne 2013; Boulton \& Tyne 2014).

9 Cette approche accorde aussi beaucoup d'importance à la diversité des situations et des locuteurs qui est indispensable pour apprendre la compétence sociolinguistique (André 2016) mais aussi la compétence socio-pragmatique que les dialogues construits ne permettent pas d'aborder en isolant les actions accomplies et en simplifiant les variables situationnelles (Alberdi et al. 2018).

Dès sa première version, le Cadre Européen Commun de Référence pour les Langues reconnaît le rôle majeur de la compétence interactionnelle dans l'apprentissage d'une langue, mais précise que la compréhension orale au niveau B1 est restreinte «à une langue standard et clairement articulée », les apprenants devant attendre le niveau B2 pour prétendre à une compréhension "même dans un environnement bruyant." (CECRL $2018: 87$ ). Nous montrerons dans la partie consacrée à l'exploitation que les interactions authentiques peuvent être comprises malgré les bruits ambiants et dans la langue telle qu'elle est réellement parlée.

\subsection{Les corpus oraux et les outils de requêtes de CLAPI}

11 La plateforme CLAPI est composée à ce jour d'une soixantaine de situations orales professionnelles et privées mettant en jeu un nombre variable de locuteurs engagés 
dans différentes activités: invitations, réunions de travail, préparation de repas, achats, consultations médicales, visites guidées, sessions de jeux, etc.

CLAPI est enrichie en continu par les nouvelles données recueillies par l'équipe suivant les travaux de recherche en cours. Il s'agit de données écologiques, c'est-à-dire qu'elles sont collectées dans leur naturalité, filmées ou enregistrées dans le lieu où elles se produisent sans aucune intervention du chercheur. Ainsi, elles se dérouleraient de la même manière en son absence, à la présence près du matériel qui se veut le plus discret possible. Aux corpus s'ajoute un ensemble d'outils de requêtes qui mettent en évidence des récurrences en articulant des outils quantitatifs et qualitatifs dans une démarche de linguistique de corpus pour identifier et illustrer certaines spécificités de l'oral dans différents contextes. S'il est difficile de rendre compte de l'ensemble des usages, nous pouvons néanmoins donner quelques exemples pertinents pour l'enseignement de l'oral suivant l'outil utilisé :

- Concordancier : afficher, écouter ou visualiser un mot ou une expression dans différents contextes dans une perspective comparative.

- Cooccurrences : relever les collocations gauches et droites d'un mot à l'oral, par exemple pour le mot trop « pas trop », " vraiment trop », « trop bien », « trop beau ».

- Lexique d'une transcription: identifier les termes les plus fréquents, notamment les marqueurs de l'oral.

- Lexique d'une forme fléchie : examiner les formes conjuguées d'un verbe courant comme « aller » ou " prendre » et les temps les plus utilisés comme le participe passé ou le présent $v s$ les moins fréquents tels que le futur, l'imparfait ou le subjonctif.

- Contexte d'emploi d'un mot : pour des usages plus avancés, repérer la position dans le tour de parole de certains marqueurs comme « ben » « mais » ou « voilà ».

Cette solution peut paraitre difficile à mettre en œuvre en situation de classe suivant le nombre d'étudiants et la durée des unités, et demande à l'enseignant un temps de préparation significatif. C'est pour ces raisons que nous avons choisi de développer un volet enseignement CLAPI-FLE en parallèle de la plateforme CLAPI, qui répond plus spécifiquement aux besoins d'enseignement avec des ressources adaptées à une utilisation en classe.

\section{L'approche méthodologique du projet CLAPI-FLE}

Pour définir ce volet enseignement, l'équipe LIS s'est entourée de spécialistes didacticiens et enseignants de français et de linguistique française, dans des départements de FLE en France et à l'étranger (Italie, Suède, Espagne, Chine, Vietnam, Liban). Des journées d'études et des séjours d'accueil au laboratoire ICAR ont été organisés avec ces partenaires afin de développer des collaborations.

Un premier projet exploratoire CLAPI-FPIE avec une équipe d'enseignants du département de Français de Lünd (Suède) a évalué la compétence d'interaction - telle qu'elle est décrite dans le CECRL - de 9 étudiants suédois apprenant le français, répartis en trois niveaux de langue A2/B1, B2 et $\mathrm{C}^{4}$, à partir d'un protocole utilisant quatre extraits de désaccords de CLAPI. Les résultats de ce projet ont démontré que les apprenants étaient capables de comprendre ces échanges dès le niveau A2/B1, en compréhension globale, et qu'ils étaient en mesure de délimiter où commençait et se terminait la séquence de désaccord, et ce malgré les différences culturelles que l'on 
peut constater dans la manifestation et la résolution d'un désaccord (Thomas et al. 2016).

Ces résultats ont confirmé la pertinence de ces données authentiques que le CECRL ne recommande qu'à partir du niveau B2. D'après ce constat, une maquette de la plateforme a été réalisée et soumise à l'évaluation de notre réseau partenaire pour en valider les fonctionnalités lors d'une journée d'études, où Isabelle Racine (Université de Genève) et Sylvain Detey (Université de Waseda) engagés dans une démarche similaire ont partagé leurs retours d'expérience sur le volet enseignement de la plateforme $\mathrm{PFC}^{5}$, Phonologie du Français Contemporain.

Sur ces bases, le site CLAPI-FLE a été conçu en proposant dans un premier temps une quarantaine d'extraits vidéo et audio, téléchargeables, contextualisés et décrits par une fiche pré-pédagogique, didactisés avec quelques pistes d'exploitation, transcrits avec différents niveaux possibles d'annotations, dotés d'un lexique et d'un dictionnaire en ligne (Etienne et Jouin 2019). Au-delà des extraits de ses corpus oraux, l'équipe LIS a souhaité créer des ressources pour l'enseignement à partir de ses travaux de recherche et de son expertise dans la description et la compréhension des interactions. C'est ainsi que deux nouvelles ressources ont été établies :

- des collections pour illustrer avec des attestations en contexte un fait de langue qu'il soit lexical, grammatical ou syntaxique ;

- des fiches explicatives ou «mini cours» pour documenter une entrée thématique de la langue (la question, les remerciements, les atténuateurs, le discours rapporté, etc.) dans toutes ses dimensions: lexicales (expressions et marqueurs), prosodiques, syntaxiques, pragmatiques, multimodales et interculturelles.

\subsection{Les extraits de corpus didactisés}

Les extraits issus des corpus de CLAPI, ont été sélectionnés par Martina Ronci, étudiante en master, dans le cadre d'une collaboration avec Florence Mourlhon-Dallies (Université Paris 5). La liste des extraits présente pour chacun d'eux un court résumé et quelques éléments de contexte, avec la possibilité de visionner en streaming cet extrait ou de le télécharger. Pour une recherche avancée, un module de sélection par facettes propose plusieurs critères : thème de conversation, format de la ressource (audio ou vidéo), domaine (privé ou professionnel), situation (réunion, repas, appel téléphonique, jeu, etc.), type de discours (formel ou informel), niveau des apprenants. Une sélection par action langagière, dont la liste a été établie à partir de référentiels par niveau (Bouquet et al. 2004) et par activité interactionnelle, vient compléter ces critères.

Chaque extrait est présenté au moyen d'une fiche pré-pédagogique qui contextualise et caractérise l'extrait selon les critères précédents, relève les principales difficultés et suggère plusieurs pistes d'exploitation en situation de classe, parfois selon le niveau des apprenants (classification A1 à C2).

Trois niveaux de transcription sont proposés: une transcription simplifiée ne comprenant que les éléments verbaux, une transcription conseillée avec certains phénomènes de l'oral annotés (pauses, chevauchements, intonations...) et une transcription personnalisée où l'enseignant choisit quelles annotations afficher. De plus, il est proposé un lexique pour visualiser le vocabulaire utilisé dans cet extrait, les mots ou formes fléchies peu fréquentes à l'oral qui demandent éventuellement une explication, un dictionnaire en ligne pour les apprenants et une synthèse quantitative 
de la variété lexicale et du débit de chacun des locuteurs. Un onglet « Extraits du même enregistrement » peut permettre de renvoyer les étudiants vers d'autres extraits d'une situation déjà expliquée en classe.

\subsection{Les collections pour illustrer certaines spécificités de l'oral}

21 En parallèle des extraits de corpus, nous avons souhaité répondre à certains des nombreux besoins exprimés par notre réseau concernant les spécificités de l'oral, relevant de la grammaire, de la syntaxe ou du lexique, que les enseignants ont des difficultés à illustrer dans différents contextes ou pour différentes fonctions pragmatiques. Nous avons proposé le terme de "collection" qui étend la notion d'exemplier à un regroupement organisé d'attestations d'un fait de langue. Ce concept de collection a d'ores et déjà soulevé un certain nombre de questions méthodologiques :

- le niveau d'explication qui accompagne les illustrations depuis une courte description du phénomène observé jusqu'à un développement plus complexe ;

- la manière d'articuler les compétences linguistiques et didactiques en proposant soit une explication produite par un interactionniste qui serait revue par un didacticien, soit une documentation délivrée par un didacticien puis relue par un linguiste ;

- la variété des situations à illustrer du point de vue du nombre de locuteurs, du format (réunion, échange commercial, consultation, repas, conversation, jeu, visite, etc.), du registre plus ou moins formel ou du canal (en présence, au téléphone ou en visioconférence);

- l'illustration des usages les plus fréquents qu'il est donc indispensable d'enseigner, sans ignorer les usages plus rares qui peuvent intéresser les apprenants d'un niveau avancé.

Les six collections à ce jour en ligne et en cours d'évaluation par les enseignants concernent les expressions ("le truc c'est que » et «c'est vrai que», la structure «tu ou vous » suivi de l'indicatif et des spécificités de la syntaxe de l'oral : «les dislocations », «le futur simple » peu fréquent à l'oral et «l'imparfait de politesse »).

\subsection{Les fiches explicatives pour documenter un fait de langue}

Dans une perspective différente allant bien au-delà de l'illustration, nous avons conçu un nouveau type de ressource, la fiche explicative, qui documente un fait de langue dans toutes ses dimensions en décrivant à la fois ses différentes fonctions pragmatiques et les procédés qui permettent d'y parvenir. Cette ressource peut s'apparenter à un mini cours avec l'ensemble des dimensions qu'elle intègre d'une part et la variété des attestations qu'elle propose d'autre part. La fiche explicative est en effet étudiée selon les axes suivants :

- lexical : expressions spécifiques, marqueurs, registre formel vs informel ;

- syntaxique : temps, structures ;

- prosodique : intonations montantes et descendantes, saillances ;

- multimodal : gestes, regards, manipulation d'artefacts ;

- interculturel : attitudes ou procédés qui pourraient surprendre un apprenant d'une autre culture, en évitant l'écueil de la stigmatisation.

Ces explications s'appuient sur les travaux de recherche et citent certains d'entre eux : un paragraphe "Pour aller plus loin» permet d'accéder à des informations plus complètes et à une courte bibliographie. Dans le cadre d'une collaboration avec Élodie 
Oursel, Université Paris 8, nous avons sélectionné avec son étudiante en master Carolina León Roa, les sujets suivants pour répondre aux attentes des enseignants en fonction de la variété de leurs objectifs mais également des niveaux de langue :

- les questions: dans une approche pragmatique qui complète la catégorisation question ouverte, fermée ou alternative enseignée dans les manuels ;

- les remerciements dont le déroulement varie beaucoup d'un pays à l'autre, même en Europe, mettant l'apprenant en difficulté dans ses échanges quotidiens, quelle que soit la situation;

- les procédés d'atténuation qui vont permettre de modérer un énoncé pour adoucir un échange tout en maintenant sa finalité ;

- le discours rapporté qu'il n'est pas toujours aisé d'identifier à l'oral et par suite de reproduire ;

- l'expression «quand même » qui modifie fréquemment un énoncé d'une manière difficile à appréhender;

- l'expression «trop » en particulier dans des formes figées très fréquentes, qui interroge dès lors qu'elle devient négative ou est associée à son contraire « peu ».

Chaque fiche explicative propose un ensemble important d'attestations où l'enseignant pourra choisir celles qui sont les plus appropriées à ses besoins. De manière familière on pourrait dire qu'il va «faire son marché » dans la variété des situations qui lui sont présentées, suivant le milieu professionnel ou privé, la situation, le nombre de locuteurs, en présentiel ou à distance. Chaque attestation est contextualisée mais aussi expliquée du point de vue des dimensions les plus pertinentes, avec une mise en relief des marqueurs, des structures ou des répétitions dans la transcription.

En détaillant l'ensemble des procédés utilisés par les locuteurs pour mener à bien leurs échanges et en exposant l'apprenant à une large variété de contexte, nous projetons que celui-ci s'appropriera petit à petit ces procédés en les comprenant in situ et par suite sera à même de les reproduire en contexte.

\section{L'utilisation des sites CLAPI-FLE et CLAPI pour l'enseignement du français}

Les données orales naturelles constituent des ressources précieuses non seulement pour le développement de la compétence linguistique des apprenants, mais surtout pour la prise de conscience du caractère culturel des productions verbales, et pour l'acquisition des structures et des principes de fonctionnement qui sont à la base de la " compétence interculturelle» préconisée par le CECRL (2018: 29, 166). En effet, l'exposition à des extraits d'échanges naturels permet à l'apprenant d'avoir accès à des éléments culturels que l'on ne trouve pas toujours dans les documents ou dialogues traditionnels. Les corpus de français parlé en interaction constituent donc des « corpus de découverte » (Holec 1990: 69) qui rendent possible l'identification des phénomènes culturellement marqués dont la maitrise est indispensable en situation de contact. Il suffit de penser, par exemple, au fonctionnement des termes d'adresse, à la formulation de l'invitation ou de la requête dans diverses langues-cultures, ou encore à des éléments culturels comme le type de nourriture préparée lors d'un repas familial, les types de fromages ou de pains que l'on peut trouver en France, les fêtes célébrées dans certaines villes françaises, etc. 
28 L'une de nous, persuadée de l'originalité et des potentialités du matériel authentique offert sur les sites de CLAPI et CLAPI-FLE, a mis en œuvre ces ressources, en tant qu'enseignante-chercheuse de Langue et Linguistique françaises dans une université italienne, pour l'apprentissage à la fois des spécificités de la langue parlée en interaction (cf. 4.1) et des phénomènes de variation (cf. 4.2), aspects qui nous semblent essentiels pour l'acquisition d'une compétence d'interaction.

Le site de CLAPI-FLE, qui propose des extraits d'interactions didactisés et adaptés en fonction des besoins et des niveaux des étudiants, a représenté notre principale ressource, alors que les données disponibles sur CLAPI, qui demandent un travail d'analyse et de sélection de la part de l'enseignant, ont permis d'enrichir parfois la description de certaines structures syntaxiques. Nous avons exploité quelques fiches explicatives du site CLAPI-FLE afin de travailler de manière plus approfondie sur des aspects particuliers de la langue parlée (cf. 4.1.1).

30 Notre exploitation de ces données orales s'insère, plus précisément, dans le cadre de l'enseignement de la Langue et de la Linguistique Françaises dans le cursus de Lingue Moderne, au Département de Lettres et Philosophie de l'Université de Trento (Italie). Les données orales naturelles ont été introduites notamment dans deux cours magistraux de Langue Française à des étudiants (italophones et francophones) de première et de deuxième année de Licence. Nous avons choisi de décrire ces deux exploitations pédagogiques pour illustrer plusieurs utilisations des ressources selon le niveau de langue, mais également en fonction des différents objectifs: compréhension, organisation des interactions, syntaxe de l'oral ou perspective interculturelle. Nous décrirons successivement ces deux exploitations avec des étudiants de première (4.1) et de deuxième année (4.2).

\subsection{Première application pédagogique avec des étudiants de première année}

31 Dans cette première mise en œuvre des ressources orales naturelles, nous avons utilisé une partie des extraits proposés sur le site de CLAPI-FLE pour illustrer des phénomènes du français parlé en interaction dans le cadre d'un cours magistral de Langue Française s'adressant à des étudiants de première année (niveaux A1-B1). Le public se compose d'un groupe hétérogène qui comprend à la fois des primo-débutants (une dizaine généralement), une majorité d'étudiants ayant un niveau B1 ou B2 (une quarantaine environ) et quelques étudiants francophones issus de l'immigration ou participant à des programmes d'accueil pour les demandeurs d'asile. Tous les étudiants de première année suivent des cours de lectorat avec des enseignants de langue maternelle et un cours magistral de Langue Française de 30 heures par semestre, qui vise à leur fournir des connaissances théoriques de linguistique (phonétique, phonologie, morphologie et syntaxe), ainsi que des notions de base du français parlé en interaction en les amenant à réfléchir aussi sur les phénomènes de variation liés à la situation de communication. L'analyse des spécificités du discours oral et notamment de la langue parlée en interaction s'insère dans la deuxième partie de ce cours magistral.

Les extraits d'interactions naturelles proposés aux étudiants ont d'abord fait l'objet d'exercices d'écoute globale portant sur la description de la situation et des activités effectuées par les participants. Cette phase d'entrée dans les données a consisté en une série de questions générales (Que font les participants ? De quoi parlent-ils ? Y a-t-il un 
problème?) susceptibles d'amener les apprenants, selon leur niveau, à décrire la situation, synthétiser le contenu de la séquence, énumérer les activités effectuées par les participants et dans certains cas, à imaginer, ou à reformuler, les propos échangés.

La deuxième étape de l'exploitation didactique des données, qui visait non seulement le développement d'une compétence linguistique mais aussi la compétence d'interaction, a permis d'étudier les spécificités générales de l'interaction orale (cf. 4.1.1), et d'approfondir certains faits de langue (cf. 4.1.2) ainsi que des micro-phénomènes linguistiques, comme les structures syntaxiques typiques de l'oral (cf. 4.1.3).

\subsubsection{L'analyse des caractéristiques générales de la langue parlée en interaction} perceptive, en se focalisant sur des aspects prosodiques particuliers, à l'aide, cette fois, d'une "transcription personnalisée » (telle que prévue par les concepteurs du site CLAPI-FLE), où nous avons choisi de ne pas faire figurer les variations de mélodie, ni la saillance des mots, phénomènes que les étudiants eux-mêmes devaient repérer et noter sur la transcription. L'objectif étant d'appliquer les notions théoriques sur l'intonation et l'accentuation à des manifestations discursives réellement attestées. Ces exercices ont favorisé la prise de conscience du rôle syntaxique de l'intonation, associée à différents types d'énoncés (assertion, question, exclamation), ainsi que la sensibilisation aux différents types d'accents (accent sémantique vs accent expressif). Nous avons ensuite distribué aux étudiants, en guise de correction, une transcription " complète ", faisant figurer les intonations et les accents d'emphase. Cette deuxième version de la transcription a constitué le document de base pour d'autres activités d'entraînement phonétique, fondées sur le repérage d'élisions ( $t$ 'as $v u$ ), de la suppression du « $e$ muet » (p'tit, s'rait, maint'nant), ou de la consonne liquide du pronom personnel « il » (i' me semble, i' faudrait). Les étudiants se sont montrés très réceptifs à la chute $\mathrm{du}$ « $\mathrm{e}$ » muet, phénomène qui produit des effets notables dans la perception du rythme de la parole, car comme l'observe Bertrand Lauret "l'effacement de "e " reconstruit la syllabe et met en contact les consonnes, constituant de nouveaux groupes inattendus qui représentent parfois des difficultés » (2007:70).

37 Ce même extrait s'est avéré intéressant pour attirer l'attention des apprenants sur les formules rituelles employées pour ouvrir une conversation téléphonique privée (que 
nous avons comparées avec celles utilisées dans des appels téléphoniques en contexte professionnel, cf. Ravazzolo et al. 2015, chapitre 6), ainsi que sur les formules utilisées pour exprimer l'invitation, activité « délicate » du point de vue relationnel, car elle met en jeu les faces des participants :

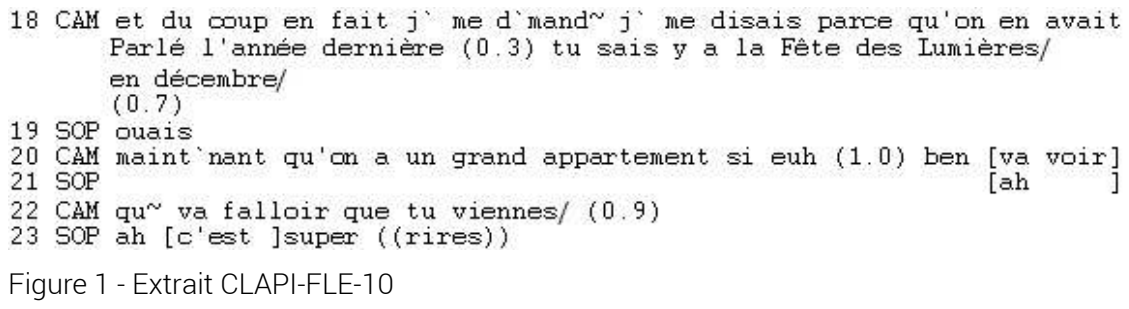

Comme l'indique le passage ci-dessus, l'invitation proprement dite, exprimée ici au moyen d'une formule injonctive ( $v a$ falloir que tu viennes), qui s'explique par la nature intime de la relation mère-fille, est précédée de marques d'hésitation, de reformulations et de justifications.

L'extrait sélectionné a été exploité ensuite pour montrer l'importance et le fonctionnement des marqueurs discursifs dans l'interaction orale. Ces petits mots, en général peu fréquents dans les dialogues pédagogiques des méthodes, prolifèrent en effet dans les conversations à l'oral et peuvent remplir de nombreuses fonctions au sein de l'interaction. À partir de l'extrait examiné, les étudiants ont été amenés à repérer ces marqueurs et à les analyser du point de vue grammatical (verbes, adjectifs, etc.) et pragmatique. Ont été mises en évidence notamment les particules ayant une valeur phatique, comme " hein » et " tu sais », ainsi que le marqueur "attends » qui retient l'attention de l'interlocuteur. Nous avons expliqué également le rôle des ponctuants comme «ben " ou des marqueurs à valeur conclusive simples (donc, voilà) et composés (donc voilà). Nous nous sommes enfin focalisée sur l'usage de «du coup ", marqueur discursif plus récent, typique de l'oral spontané, qui indique souvent un lien sémantique de cause à effet. Cette expression, « intéressante du fait de sa fréquence et de la difficulté d'interprétation qu'elle représente pour les apprenants non francophones " (Groupe ICOR, Guinamard 2015: 147), mériterait d'être traitée de manière approfondie, afin d'aider les étudiants à en comprendre les valeurs sémantiques et conversationnelles lorsqu'ils se trouveront en situation d'immersion.

Pour étudier les aspects multimodaux de l'interaction et les phénomènes de collaboration discursive, nous avons ensuite sélectionné d'autres extraits vidéo, comme par exemple CLAPI-FLE-29, Les pièces d'un hôtel (cf. figure 2). Cet extrait, tiré d'une réunion d'architectes qui discutent de la réhabilitation d'un château en centre de séminaires, a contribué par ailleurs à enrichir le lexique des apprenants dans le domaine du français du tourisme (dont l'étude est prévue dans les cours de lectorat), en apportant des données naturelles qui intègrent les documents pédagogiques fournis par leur manuel de référence.

41 L'extrait Les pièces d'un hôtel révèle la manière dont les actions gestuelles et la manipulation d'objets contribuent à la construction des activités. L'analyse de l'extrait du point de vue interactionnel a été précédée d'une explication sur les différents types de gestes communicatifs utilisés dans la communication orale (cf. Ravazzolo et al. 2015 : 36-39). Nous avons relevé en particulier la fréquence des gestes déictiques, utilisés par les participants pour désigner sur le plan les référents mentionnés dans le discours (les parkings d'attente, la zone de desserte, l'accès principal, l'accès de service, etc.), ou pour 
revenir sur un élément du discours déjà énoncé, comme c'est le cas lorsque l'un des locuteurs, $\mathrm{L}$, pointe du doigt le plan qui avait déjà été mis de côté par $\mathrm{C}$ dans l'énoncé de $\mathrm{L}$ (ligne 20 , « et y avait une autre chose aussi à expliquer ») où il associe clairement un geste déictique au moyen du bras et de l'index pointé vers le "vieux " plan et suivi d'un geste iconique mimant la forme des parkings dessinés sur le plan («c'était l'idée des parkings en fait »). Dans d'autres cas, les gestes déictiques peuvent servir pour formuler des demandes d'identification de quelques éléments graphiques du plan:

$26 \mathrm{M}$ [ et ] ça c'est quoi là/ euh ce mur/ $\backslash$ c'est [un mur/ ]

Figure 2- Extrait CLAPI-FLE-29

\subsubsection{La description de faits de langue à l'aide des fiches explicatives}

L'enseignement des caractéristiques de la langue parlée a nécessité l'approfondissement de quelques phénomènes de nature pragmatique souvent représentés dans les extraits analysés avec les étudiants. Ainsi l'observation de certaines activités interactionnelles (comme l'offre ou l'invitation) a-t-elle imposé une analyse détaillée des ressources mises en œuvre par les locuteurs dans la production de leurs énoncés. Nous nous sommes intéressée, par exemple, aux procédés d'atténuation employés, dans une perspective socio-relationnelle, pour inter-agir dans le respect des autres et de soi-même. La fiche explicative « Les atténuateurs » disponible sur le site de CLAPI-FLE a constitué une excellente ressource pour aborder ce sujet. Après avoir défini la notion d'atténuation en relation avec la théorie de face work de Goffman (1973), nous avons exposé les quatre procédés d'atténuation illustrés dans la fiche: (i) les modalisateurs, (ii) les formules de politesse, (iii) l'explication/l'argumentation, (iv) la blague. Chaque procédé, assorti d'un exemple choisi parmi ceux qui sont proposés dans la fiche, a fait l'objet d'une étude approfondie des ressources multimodales qui le caractérisent. L'extrait sélectionné (1.5, figure 3) pour documenter le premier procédé (les modalisateurs) a permis par ailleurs d'attirer l'attention des apprenants sur un aspect culturel lié à la formulation de l'offre, à savoir l'emploi de l'adjectif « petit(e)» qualifiant l'aliment ou la boisson proposé(e)/demandé(e) : « vous voulez un petit jus de fruit/ ", « je prendrai un petit whisky »). Loin de pouvoir être interprétée littéralement, cette unité linguistique, qui connaît des variations mélodiques liées au contexte et aux intentions du locuteur, constitue une stratégie de minimisation susceptible d'adoucir l'offre ou la requête.

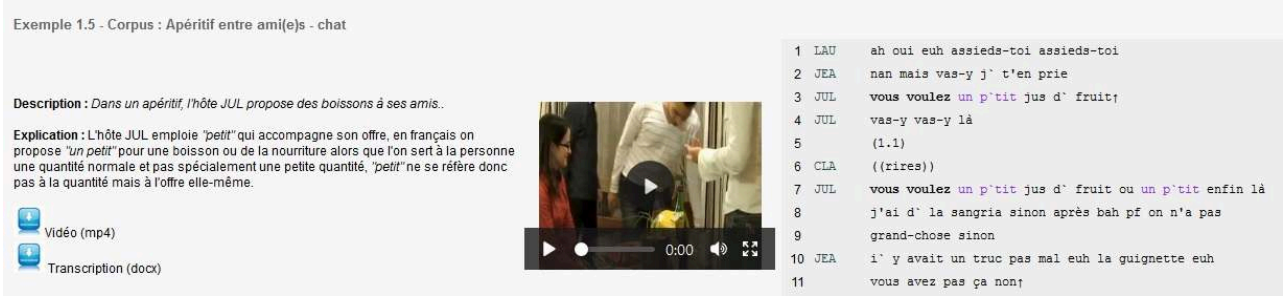

Figure 3 - Fiche Atténuation, Exemple 1.5, Corpus apéritif Chat

L'extrait (3.2) sélectionné pour illustrer le procédé atténuateur « argumentation » met en évidence l'emploi de la construction "oui non mais", introduisant un contreargument et signalant un enchaînement non préféré. La présentation de cette construction particulière, fondée sur le cumul de marqueurs discursifs antinomiques, a été complétée par une analyse détaillée proposée sous forme de citation dans la 
rubrique « Pour aller plus loin ». Ainsi, comme le montre André (2006), la formule « oui non mais", souvent suivie de "attends/attendez", peut être analysée comme la construction progressive d'un point de vue divergent :

Le oui informe les interlocuteurs que les informations qu'ils viennent de communiquer ont été comprises et sont prises en compte. Le non indique que le locuteur n'est pas d'accord avec ces mêmes informations. Enfin, le bloc mais attends, également fréquemment rencontré dans des situations de conversations quotidiennes, communique aux participants que le locuteur L1 est en possession d'éléments supplémentaires qu'il va apporter. (André 2006 : 419)

Une fois terminée la présentation des atténuateurs et de leurs procédés, nous avons soumis aux étudiants les autres exemples proposés dans la fiche (extrait vidéo et transcription) en leur demandant d'identifier et analyser les différents procédés d'atténuation mis en place par les locuteurs.

La fiche explicative, avec ses nombreux extraits commentés et les références bibliographiques conseillées, offre donc la possibilité de développer la présentation structurée et documentée de phénomènes linguistico-pragmatiques, souvent marginalisés dans les manuels pédagogiques, en bénéficiant des explications et des analyses des concepteurs.

\subsubsection{Les structures syntaxiques typiques de l'oral}

L'étude des structures typiques de la langue parlée s'est focalisée ensuite sur l'analyse de constructions syntaxiques particulièrement récurrentes à l'oral, à savoir les dislocations, à gauche et à droite, les constructions clivées et pseudo-clivées. L'explication théorique de certains types de construction a bénéficié de l'apport de nombreux extraits du site CLAPI-FLE, et d'autres micro-extraits que nous avons sélectionnés à partir des corpus de la banque de données CLAPI. Pour la présentation des dislocations nous avons consulté en outre la collection « Les dislocations».

\section{a) Les constructions disloquées}

47 La dislocation, qui se caractérise par le détachement d'un élément en tête ou en fin de phrase, repris ou annoncé par un pronom personnel ou démonstratif, a été traditionnellement (Riegel, Pellat, Rioul 1994 : 425) considérée comme une forme de mise en relief. Or, s'il est vrai que les dislocations permettent de marquer l'élément saillant de la phrase, leur rôle ne peut toutefois pas être réduit à une forme de focalisation, du moins au niveau conversationnel. En effet, ces structures ont un rôle cognitif dans l'organisation et la planification du discours et, peuvent accomplir des fonctions diverses (Horlacher 2015, Ravazzolo et al. 2015 : 94-97) dans l'interaction.

48 À partir de la collection sur les dislocations, nous avons sélectionné les extraits susceptibles d'illustrer les catégories d'analyse suivantes: la nature des constituants détachés, la fonction syntaxique des éléments disloqués, leurs fonctions. Par rapport à l'organisation de la collection, nous avons modifié la présentation de ces structures, en distinguant nettement la dislocation à gauche de la dislocation à droite, et nous avons enrichi la description des fonctions pragmatiques de ces constructions, grâce à l'examen d'autres corpus de CLAPI et aux résultats de recherches antérieures (cf. Ravazzolo et al. 2015, chapitre 5.3.). 


\section{a-1) Les dislocations à gauche} sous forme de reprise pronominale. Après avoir distingué les constructions selon la nature pronominale ou nominale du constituant détaché, nous avons attiré l'attention des apprenants sur les fonctions syntaxiques de l'élément disloqué à gauche (sujet, COD, COI). L'analyse des extraits a révélé qu'il est très souvent le sujet de la phrase et les étudiants ont eux-mêmes observé la fréquence des formes de redoublement pronominal à l'oral: «moi je», «toi tu», «nous on", ainsi que la fréquence des formes : nom propre + pronom personnel, comme dans l'exemple ci-dessous :

FLO euh [mathilde] elle fait euh sciences de l'éducation

Figure 4 - Corpus CLAPI, Repas entre ami(e)s Olives

Pour ce qui est des fonctions, le volet pragmatique de la collection sur la dislocation met en avant l'attribution d'une saillance à l'élément disloqué, que ce soit pour gérer le temps d'élaboration du discours ou bien pour faciliter le décodage chez l'interlocuteur par un travail de clarification et de désambiguïsation. Nous avon
gauche

1) Ces constructions contribuent en particulier à renforcer la position énonciative du locuteur ou à ménager son temps de parole pour différentes raisons :

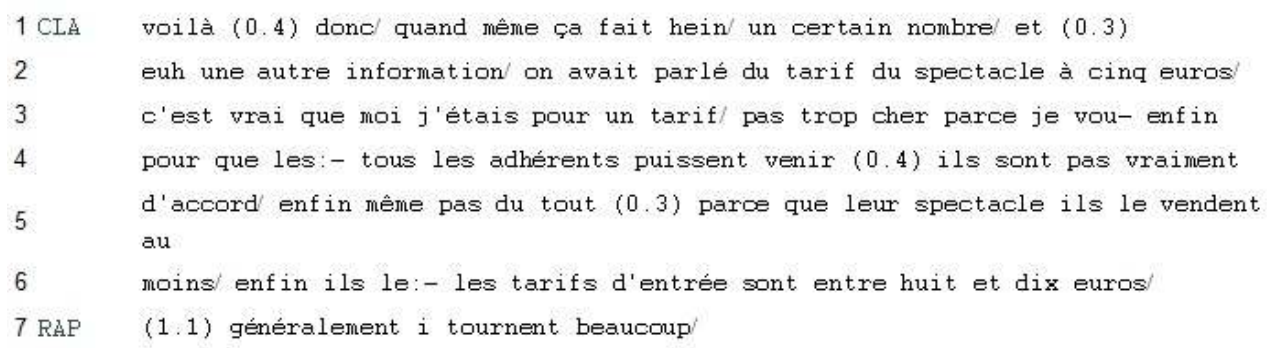

Figure 5- Collection CLAPI-FLE Exemple 7.3 Corpus Réunion organisation événement

Dans le passage ci-dessus, l'emploi de la dislocation "pronom tonique + pronom clitique " vise à renforcer le point de vue du locuteur, alors que la deuxième dislocation à gauche «leur spectacle ils le vendent [...]» est employée pour retarder l'annonce "délicate» du prix du spectacle. L'effet dilatoire de cette formule est renforcé par l'inachèvement (enfin ils le:-) et la reformulation (les tarifs d'entrée sont [...]).

2) Les locuteurs utilisent également cette structure pour (ré-) introduire un nouveau thème, un nouvel argument ou un nouvel exemple :

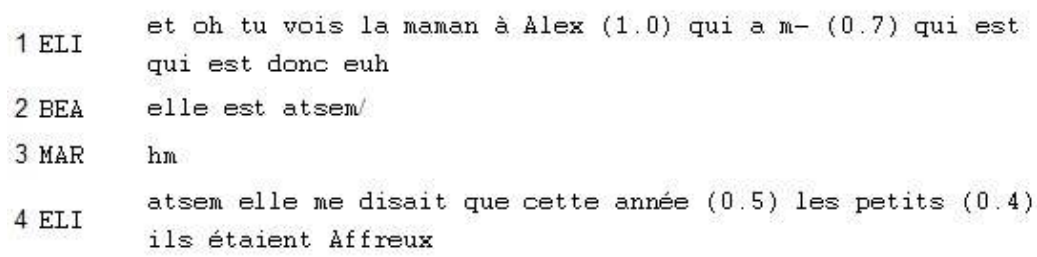

Figure 6- Collection CLAPI-FLE Exemple 3.1 Corpus Repas Kiwi 
Dans cet extrait on trouve deux dislocations : la première (« la maman à Alex [...] elle ») sert à introduire un nouvel objet de discours, relié au thème principal des expériences à l'école maternelle et à orienter l'attention des interlocutrices vers une personne spécifique ; la deuxième dislocation permet à ELI d'attirer l'attention sur le nouveau thème de la phrase « les petits » avant de faire suivre la prédication. Grâce à une écoute perceptive de cet extrait, focalisée sur le repérage des accents d'emphase et des pauses, les étudiants ont pu comprendre, entre autres, comment la saillance se construit, au niveau syntaxique, mais aussi au niveau prosodique.

3) La dislocation à gauche peut marquer aussi une mise en contraste, comme dans l'exemple suivant, où le redoublement pronominal sert à souligner ce que chacun fait par rapport aux autres :

\begin{tabular}{|l|l|l|}
\hline 1 & JUL & noël en fait vous avez fêté ensemble $\uparrow$ \\
\hline 2 & & $(1.4)$ \\
\hline 3 & ANN & ben [euh \\
\hline 4 & ROM & --_ [moi je retourne chez mes parents \\
\hline 6 & ANN & ouais moi je fais pareil hein \\
\hline 7 & JUL & [((rires)) \\
\hline 8 & ANN & [mais j'habite pas ici donc euh \\
\hline 9 & & $(0.4)$ \\
\hline 10 & ANN & c'est loin quoi \\
\hline 11 & JUL & hm hm \\
\hline 12 & ROM & et toi tu vas où $\uparrow$ \\
\hline 13 & JUL & BEN moi en fait c- c'est un petit peu la même chose \\
\hline 14 & & en fait moi je vais aller voir mon frère Timothée \\
\hline 15 & & il va rentrer à l'île de la réunion \\
\hline
\end{tabular}

Figure 7 - Collection CLAPI -FLE. Exemple 7.5 Corpus Apéritif entre ami(e)s pois

\section{a-2) Les dislocations à droite}

Dans ces constructions, on détache un élément en fin de phrase et on l'anticipe par un pronom. Dans ce cas, le thème vient après le noyau verbal.

Après avoir analysé les fonctions syntaxiques et la nature des constituants détachés à droite nous avons présenté des micro-extraits et d'extraits où la disloquée à droite semble accomplir des fonctions spécifiques au niveau pragmatique :

a. une fonction de désambiguïsation et de clarification de l'antécédent pronominal ; 
b. une fonction dans la gestion des thèmes, surtout dans des contextes qui comportent une évaluation d'un objet de discours (cf. ex. 1.3), comme dans l'exemple suivant :

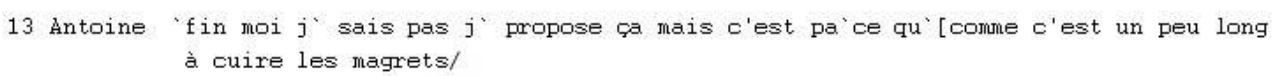

\section{b) Les constructions clivées} avec la description des pseudo-clivées, constructions qui s'articulent en deux parties :

Ces constructions donnent au locuteur la possibilité d'encadrer un élément de son énoncé au moyen de la structure «c'est qui/c'est que » et d'attirer donc l'attention de l'interlocuteur sur cet élément particulier.

Pour l'illustration des clivées (et des pseudo-clivées) nous avons retenu des exemples de CLAPI-FLE et de CLAPI.

Le premier exemple, tiré de l'extrait CLAPI-FLE-13, a été exploité pour analyser le fonctionnement de cette construction syntaxique dans le contexte d'une visite guidée, situation dans laquelle les clivées sont massivement employées pour attirer l'attention des visiteurs sur des informations spécifiques : nom d'artistes ou d'auteurs des objets, datation des objets, etc. (cf. figure 9).

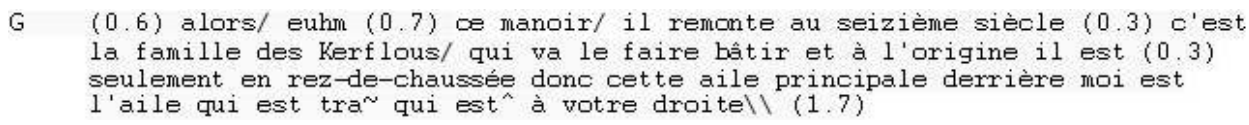

2 D'autres extraits de CLAPI ont été soumis aux étudiants pour faire observer les fonctions syntaxiques les plus fréquentes (sujet, COD) de l'élément encadré. Par exemple :

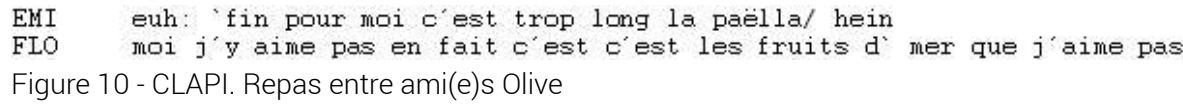

3 Quant aux fonctions pragmatiques, outre le rôle de focalisation et de hiérarchisation des informations lié à des visées pédagogiques, nous avons montré l'usage de la clivée dans des contextes où il s'agissait d'introduire un élément s'opposant à un point de vue précédent ou corrigeant un tour de parole ou un segment antérieur.

\section{c) Les constructions pseudo-clivées}

- une première partie constituée d'un syntagme nominal (ex. "le problème c'est [...]») ou d'une proposition («ce que je veux c'est [...] »)

- une deuxième partie qui fournit l'information essentielle.

Avec l'analyse de ces exemples, les étudiants ont pu constater que ce type de dispositif syntaxique est particulièrement utilisé quand on exprime un point de vue ou quand il faut argumenter, et qu'il comporte souvent, dans sa première partie, des formules quasiment figées, étant donné leur fréquence, telles que «le problème c'est que » ou «le truc c'est que », variante familière qui fait d'ailleurs l'objet d'une collection : 


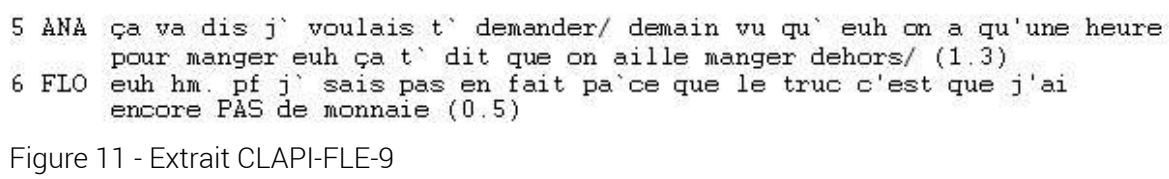

La présentation théorique des différentes structures syntaxiques de la langue parlée et l'analyse des extraits ont été suivies d'une phase d'application. Les étudiants ont été divisés en groupes de 6, et chaque groupe a reçu une transcription de CLAPI-FLE ou de CLAPI (ainsi que des extraits d'interaction d'autres corpus). Après avoir présenté les différents enregistrements, nous avons demandé à chaque groupe de repérer les différentes structures syntaxiques utilisées dans l'extrait examiné et de les décrire du point de vue formel et pragmatique. Au terme de ce travail, 4 groupes sur 6 ont relevé la totalité des structures syntaxiques présentes dans l'extrait, alors que 2 groupes en ont relevé la majorité ( 3 sur 5,4 sur 6 ). Tous les groupes ont pu analyser de manière pertinente l'usage de ces dispositifs syntaxiques en faisant preuve donc d'une certaine progression dans leur compréhension orale d'extraits naturels de langue parlée. Cette activité, ainsi que la mise en commun des résultats de chaque groupe, a favorisé la prise de conscience de la fréquence de ces structures syntaxiques dans tout type de discours oral, et contribué, comme nous l'espérons, à l'élimination des préjugés concernant le caractère familier de ces constructions.

\subsection{Deuxième application pédagogique avec des étudiants de deuxième année}

Une partie des extraits proposés sur le site de CLAPI-FLE et d'autres extraits de CLAPI ont été utilisé afin d'aborder la problématique de la variété des genres discursifs de l'oral dans le cadre d'un cours magistral de Langue Française s'adressant à une quarantaine d'étudiants italophones et francophones de deuxième année (niveau B1B2) du cursus de Lingue Moderne (Département de Lettres et Philosophie de l'Université de Trento). L'emploi de ces ressources a permis d'illustrer les spécificités de quelques types d'interactions et d'activités discursives et interactionnelles qui les caractérisent. L'exploitation didactique des données naturelles a donc été mise au service du développement de la « compétence interactionnelle » (Pekarek Doehler 2005, 2006), qui renvoie à la capacité des locuteurs à s'adapter aux variations situationnelles, grâce à la mobilisation de ressources linguistiques, discursives, sociolinguistiques et interactionnelles. Il s'agit plus précisément de savoir adapter son discours (et son comportement) aux différentes situations de la vie quotidienne et de mobiliser pour chaque situation de communication des ressources appropriées (registre de langue, termes d'adresse, routines et formules figées, manière d'introduire ou de clore un thème, enchaînement habituel des actions, structure d'une activité, etc.). Cela présuppose que l'on identifie plusieurs types d'interactions qui se différencient selon les locuteurs, le lieu, la durée, la finalité, mais aussi selon d'autres traits comme le caractère privé/public/professionnel de l'interaction, ou son caractère médiatisé (en face à face, par téléphone, par écran).

$\mathrm{Au}$ niveau théorique, la classification des genres discursifs de l'oral proposée se fonde sur le modèle de Kerbrat-Orecchioni et Traverso (2004) qui distinguent un premier niveau générique constitué des types d'interactions et un deuxième niveau qui se compose des différents types d'activités discursives. 
69 L'analyse a porté sur 5 types d'interactions : (i) l'interview et (ii) le débat médiatiques, (iii) l'interaction de commerce, (iv) la visite guidée, et (v) la conférence (la conversation ordinaire avait été déjà abordée en première année). L'objectif était de montrer le mode de structuration des échanges et des activités, ainsi que la co-construction à plusieurs des actions sociales et leur caractère situé, lié à des pratiques spécifiques, réalisées dans un contexte socio-culturel précis.

70 Ainsi les étudiants ont-ils été amenés à observer, par exemple, comment se réalisent les séquences d'ouverture et de clôture dans les différentes situations. En effet, bien que ces séquences liminaires soient extrêmement importantes au niveau rituel et relationnel, elles «sont souvent réduites à une ou deux répliques dans les dialogues pédagogiques des méthodes » (Ravazzolo et al. 2015 : 121).

71 Les ressources de CLAPI-FLE et de CLAPI ont été exploitées notamment pour étudier l'interaction dans les commerces et la visite guidée.

\subsubsection{Les interactions dans les commerces}

72 Pour aborder les caractéristiques principales de l'interaction dans un commerce, nous avons sélectionné des extraits d'interactions se déroulant dans une boulangerie et dans une fromagerie françaises. En particulier, nous avons soumis aux étudiants deux extraits de CLAPI-FLE (CLAPI-FLE-1 « Achat rapide », CLAPI-FLE-2 « Série d'achats ») et deux extraits de CLAPI (Interactions commerciales - Fromagerie).

Grâce à ces ressources, nous avons travaillé à la fois sur des aspects liés à l'organisation et à la gestion de l'interaction, ainsi que sur la notion d'activité interactionnelle et d'action langagière (en accord avec la perspective actionnelle privilégiée dans le CECRL, qui considère l'apprenant comme un acteur social ayant à accomplir des tâches).

74 Après avoir dégagé le script interactionnel commun aux différentes transactions, c'està-dire l'enchaînement habituel des activités qui caractérisent l'interaction commerciale, les étudiants ont noté les divergences que l'on pouvait enregistrer dans les quatre situations examinées. En effet, si le premier extrait ("Achat rapide») correspond au déroulement prototypique de l'interaction dans un commerce, les autres extraits présentent des particularités qui complexifient le script de base : présence de séquences descriptives, négociations concernant la sélection du produit, séquences conversationnelles avec des clients habituels, etc.

Dans un premier moment, nous avons travaillé sur la réalisation d'activités interactionnelles spécifiques dans ce contexte, comme la requête. Les étudiants ont repéré les diverses formes de réalisation de la requête dans les extraits examinés : du simple syntagme nominal (ex. "une flûte et une baguette courte ", " un pain au chocolat ») ou du simple geste de poser le produit sur le comptoir, à une formulation plus complexe comme l'assertion de l'action d'acheter, exprimée principalement au futur proche ( «bah je vais vous prendre déjà un bout de comté fruité »; " ensuite je vais prendre un [...]», " on va tester un langres »). Ces réalisations ont été comparées avec d'autres formulations qui ne figuraient pas dans les documents analysés, mais qui sont néanmoins souvent utilisées dans les petits commerces français, comme par exemple l'assertion d'un désir accompagnée de formes verbales de politesse: «je voudrais », « j'aurais voulu », « je voulais », etc. (cf. Ravazzolo et al. 2015 : 137).

Dans cette perspective, l'extrait CLAPI-FLE-2 « Série d'achats » (cf. figure 12) s'est avéré particulièrement intéressant pour travailler sur une modalité particulière de 
formulation de la requête : après avoir choisi des produits librement accessibles dans le magasin, une cliente donne le sac qui les contient à la vendeuse afin qu'elle puisse en calculer le prix :

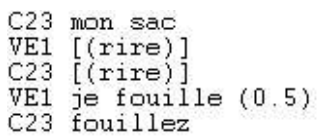

Figure 12 - Extrait CLAPI-FLE-2

77 Dans ce cas, la réalisation de la requête et son exécution se fondent prioritairement sur la dimension gestuelle : la vendeuse sort les produits, les pèse et verbalise le prix; elle peut éventuellement formuler des commentaires sur les produits (« vous avez encore des mouches $\uparrow »)$, mais ne les mentionne pas systématiquement. Pour comprendre ce qui se passe dans cet extrait, la vidéo est donc fondamentale. En contexte commercial, la réalisation des activités repose donc sur l'articulation entre actions langagières et actions non langagières. Le geste peut parfois être indispensable dans la sélection d'un produit et remplacer l'expression verbale de la quantité. Par exemple, dans un extrait du corpus « Fromagerie » de CLAPI, la vendeuse se sert uniquement du geste déictique pour avoir des indications sur la quantité de fromage qu'elle doit couper.

Enfin, au niveau lexical, les extraits sur les petits commerces se sont révélés d'une grande richesse en raison de la présence de plusieurs termes désignant des types de pain ou de fromage français. Ces culturèmes, unités linguistiques porteuses d'informations culturelles, considérés comme les «symptômes de la différence des langues » (Cassin $2014: 235$ ), ont suscité l'intérêt des étudiants.

\subsubsection{La visite guidée}

79 La visite guidée constitue un genre discursif et interactionnel qui contient de nombreux sous-genres selon les objets, les lieux, la finalité. Pour présenter les caractéristiques principales de ce type d'interaction nous avons exploité des extraits de la visite guidée d'un manoir (site CLAPI-FLE) et des extraits de visites guidées réalisées à l'intérieur d'un musée (corpus « Saint-Denis »).

Les extraits de CLAPI-FLE (CLAPI-FLE-13 « Début de visite guidée », CLAPI-FLE-14 « Les superstitions au XIX siècle », CLAPI-FLE-15 « La fin de la visite guidée ») ont été soumis aux étudiants pour illustrer d'abord la modalité de réalisation des séquences d'ouverture et de clôture de la visite, qui a été ensuite comparée aux séquences liminaires d'autres types d'interaction analysées précédemment.

81 L'objectif était de se concentrer sur le discours produit par le guide, afin de dégager les principales stratégies mises en œuvre dans la transmission du savoir. L'analyse des extraits vidéo et de la transcription a conduit à l'identification de procédés multimodaux dans l'introduction des éléments faisant l'objet d'une explication ou d'une description. Comme le mettent en évidence les extraits de CLAPI-FLE, le guide oriente le regard des visiteurs vers l'objet de référence grâce à la mobilisation de ressources linguistiques et gestuelles :

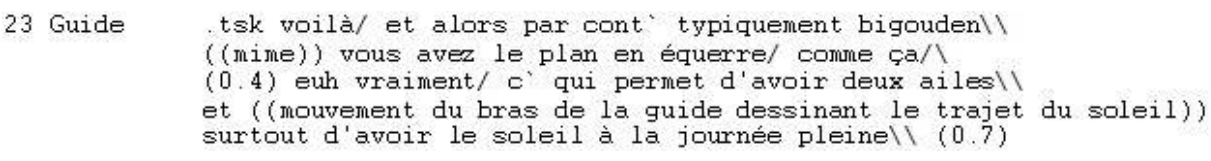

Figure 13 - CLAPI-FLE-13 

des petites dimensions du lit qu'elle vient de désigner aux visiteurs. La séquence
explicative est ici signalée par l'emploi de mots et marqueurs spécifiques (deux raisons, parce que, pourquoi, donc) :

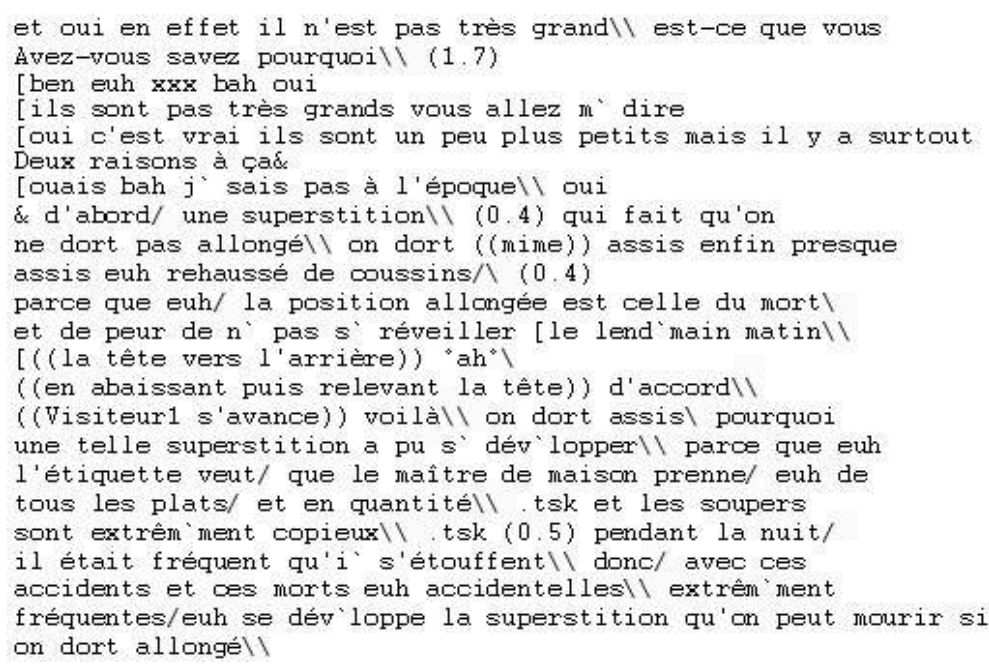

Figure 14 - Extrait CLAPI-FLE-14

Les étudiants ont ensuite pu repérer les stratégies mises en œuvre par la guide afin de faciliter la réception, la compréhension et l'assimilation des informations. Parmi les ressources mobilisées à cette fin, ils ont souligné en particulier le rôle du questionnement. Dans les extraits de CLAPI-FLE (cf. figure 15), en effet, la guide pose des questions qui semblent interroger les visiteurs mais qui en réalité constituent une stratégie de focalisation de l'attention collective :



Figure 15 - Extrait CLAPI-FLE-13

86 Ces questions, typiquement utilisées en contexte didactique, introduisent les unités de transmission des connaissances et fonctionnent comme des phatiques, susceptibles d'attirer l'attention des visiteurs sur les informations saillantes.

Les étudiants ont enfin réfléchi au rôle des visiteurs dans les interactions examinées, à travers l'analyse de leurs contributions verbales et gestuelles. Ainsi ont-ils été amenés à 
identifier les indices de leur collaboration, que ce soit à travers la production de régulateurs, de marqueurs de surprise, de commentaires sur les objets exposés (cf. par exemple le commentaire du visiteur sur la petitesse du lit, dans CLAPI-FLE-14, qui suscite le rire partagé) ou sous forme de co-énonciation, comme dans l'extrait suivant, où le visiteur (ligne 7) complète l'énoncé de la guide en anticipant une information sur le toit du bâtiment :

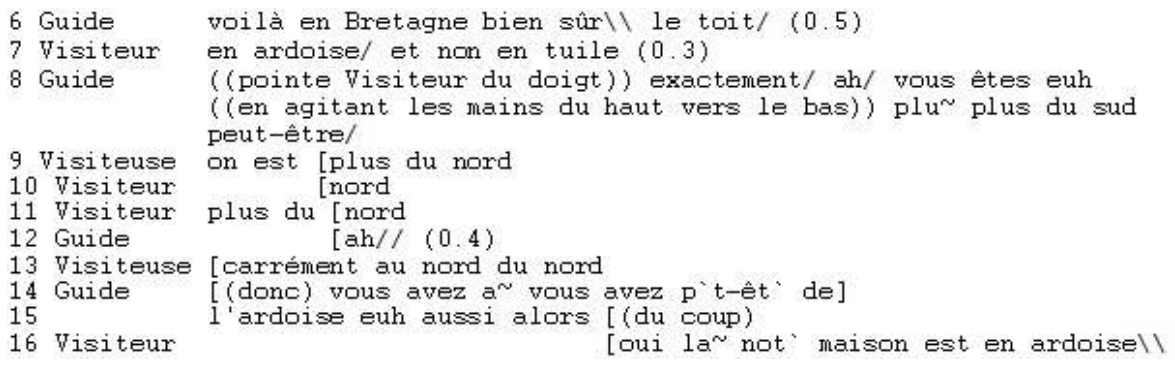

Figure 16 - Extrait CLAPI-FLE-13

Ces extraits d'interactions orales naturelles nous ont permis de sensibiliser les apprenants aux normes sociales qui régissent les interactions dans la langue-culture française: qu'il s'agisse des règles de politesse, des normes qui déterminent le fonctionnement des rituels, ou encore des règles liées à des scénarios ou à des genres interactionnels spécifiques.

Les exploitations pédagogiques réalisées grâce aux données de CLAPI et de CLAPI FLE montrent la richesse des extraits d'interactions naturelles et la variété d'utilisations possibles. Dans un souci de simplification, nous synthétisons dans le tableau ci-dessous les différents types d'activités proposées en classe, selon l'objectif d'apprentissage, les ressources et le niveau des étudiants.

\begin{tabular}{|l|l|l|l|}
\hline $\begin{array}{l}\text { Objectifs } \\
\text { d'apprentissage }\end{array}$ & Activités & Ressources & Niveaux \\
\hline Compréhension orale & $\begin{array}{l}\text { écoute/ visionnage de l'extrait : } \\
\text { questions ouvertes résumé de la } \\
\text { séquence; identification de } \\
\text { problèmes/difficultés }\end{array}$ & CLAPI FLE-10 \\
CLAPI FLE-29 & A1-B1 \\
\hline $\begin{array}{l}\text { Analyse de la structure } \\
\text { de l'interaction }\end{array}$ & $\begin{array}{l}\text { écoute/visionnage de l'extrait : } \\
\text { identification des activités } \\
\text { effectuées par les participants }\end{array}$ & $\begin{array}{l}\text { CLAPI FLE-10 } \\
\text { CLAPI FLE-29 }\end{array}$ & A1-B1 \\
\hline $\begin{array}{l}\text { Repérage/perception de } \\
\text { phénomènes } \\
\text { multimodaux } \\
\text { (prosodiques et gestuels) }\end{array}$ & $\begin{array}{l}\text { écoute/visionnage avec la } \\
\text { transcription personnalisée : } \\
\text {-notation des pauses, des variations } \\
\text { le mélodie, de l'intensité, de } \\
\text { l'accent expressif; l'extrait 29: observation des } \\
\text { gestes, description et analyse de } \\
\text { leurs fonctions interactionnelles }\end{array}$ & $\begin{array}{l}\text { CLAPI FLE-10 } \\
\text { CLAPI FLE-29 }\end{array}$ & A1-B1 \\
\hline
\end{tabular}




\begin{tabular}{|c|c|c|c|}
\hline $\begin{array}{l}\text { Auto-évaluation et } \\
\text { identification de } \\
\text { phénomènes } \\
\text { phonétiques et } \\
\text { linguistiques spécifiques }\end{array}$ & $\begin{array}{l}\text { (4) écoute/visionnage à l'aide d'une } \\
\text { transcription « complète » : } \\
\text { - correction de l'activité } \\
\text { précédente } \\
\text { - repérage d'élisions, du « e » caduc, } \\
\text { etc. } \\
\text { - identification de formes rituelles } \\
\text { spécifiques (CLAPI FLE-10) } \\
\text { - lexique de spécialité (CLAPI } \\
\text { FLE-29) } \\
\text { - observation et analyse des } \\
\text { marqueurs discursifs }\end{array}$ & $\begin{array}{l}\text { CLAPI FLE-10 } \\
\text { CLAPI FLE-29 }\end{array}$ & A1-B1 \\
\hline $\begin{array}{l}\text { Analyse des structures } \\
\text { syntaxiques typiques de } \\
\text { l'oral }\end{array}$ & $\begin{array}{l}\text { Observation, description et } \\
\text { repérage : la dislocation à droite, la } \\
\text { dislocation à gauche, les } \\
\text { constructions clivées, les } \\
\text { constructions pseudo-clivées }\end{array}$ & $\begin{array}{l}\text { Collections CLAPI FLE } \\
(3.1,7.3,7.5) \text {, CLAPI FLE } \\
(9,13) \text { et CLAPI (ex. } \\
\text { repas entre amies) }\end{array}$ & A1-B1 \\
\hline $\begin{array}{l}\text { Développement d'une } \\
\text { compétence } \\
\text { interactionnelle }\end{array}$ & $\begin{array}{l}\text { Sensibilisation aux variations } \\
\text { situationnelles: } \\
\text { - analyse de la structure globale } \\
\text { de différents types d'échanges } \\
\text { (interactions dans les } \\
\text { commerces, visites guidées, } \\
\text { etc.) } \\
\text { - identification et description } \\
\text { des activités interactionnelles; }\end{array}$ & $\begin{array}{l}\text { CLAPI FLE-1, 2,13, 14, } 15 \\
\text { CLAPI (« Fromagerie ») } \\
\text { Corpus « Saint -Denis» }\end{array}$ & B1-B2 \\
\hline $\begin{array}{l}\text { Acquisition } \\
\text { compétence } \\
\text { interculturelle }\end{array}$ & $\begin{array}{l}\text { comparaison interculturelle: } \\
\text { • actions langagières (ex. } \\
\text { formulation de la requête ou } \\
\text { de la question), } \\
\text { - termes d'adresse } \\
\text { - formules rituelles et de } \\
\text { politesse, } \\
\text { - éléments culturels (nourriture, } \\
\text { fêtes célébrées, etc.) }\end{array}$ & $\begin{array}{l}\text { CLAPI FLE }(10,29,1,2,9 \text {, } \\
13) \\
\text { CLAPI }\end{array}$ & A1-B2 \\
\hline
\end{tabular}

\section{Conclusions}

Les exploitations des données orales naturelles dans l'enseignement d'une langue et culture étrangères se sont révélées particulièrement utiles avec les étudiants de l'Université de Trente, pour différents objectifs d'apprentissage et différents niveaux de compétence, depuis la formulation d'une invitation en contexte informel jusqu'à l'assimilation d'unités linguistiques (les marqueurs discursifs) et de structures syntaxiques plus difficiles à manipuler en situation d'interaction. Dans cette perspective, les extraits du site CLAPI-FLE répondent à cette palette de besoins en 
raison de leur forme didactisée qui facilite leur sélection et de la possibilité de modifier la granularité des transcriptions. Le travail effectué avec les étudiants a montré que l'exposition à ces échantillons de langue parlée contribue à améliorer leur compréhension de l'oral et à développer une compétence interactionnelle. En effet, l'exposition à ces données recueillies dans différentes situations familiarise l'apprenant à des particularités de prononciation et à des formes prosodiques ou syntaxiques peu représentées dans les dialogues pédagogiques. Ainsi peut-on créer des habitudes d'écoute qui amènent les apprenants à découvrir des manières de dire moins conventionnelles et à se repérer dans des productions où les groupes rythmiques diffèrent $d u$ découpage syntaxique ordinaire. Travailler avec des échantillons d'interactions naturelles sensibilise également les étudiants aux phénomènes de variation situationnelle et culturelle et facilite l'acquisition des normes qui régissent chaque type d'interaction. Grâce à la diversité des données, les apprenants peuvent donc développer une compétence susceptible de les conduire à s'exprimer de manière appropriée dans différents contextes. La prise de conscience du caractère culturel des productions verbales favorise par ailleurs l'acquisition de la «compétence interculturelle » (CECRL 2018 : 166) préconisée par le CECRL, car les étudiants peuvent relever des phénomènes culturellement marqués dont la maitrise est indispensable en situation de contact.

91 L'expérience de l'application pédagogique des ressources de CLAPI-FLE avec nos étudiants a révélé néanmoins la nécessité d'une exposition régulière à ce type de données, car, au début de ce parcours de découverte, les étudiants, habitués à travailler prioritairement avec des dialogues pédagogiques ou des extraits d'interactions médiatiques, ont rencontré des difficultés de compréhension et de perception. La possibilité d'écouter un nombre toujours croissant d'enregistrements naturels les a conduits à se familiariser avec des rythmes de parole «nouveaux » et à développer des stratégies de compensation susceptibles d'améliorer leur niveau de compréhension, dans le but non pas de discriminer toutes les formes, mais de faire des prévisions et de les vérifier en puisant des indices sur le matériel sonore et visuel (cf. Holec 1990: 71-73.).

92 Le retour des apprenants s'est avéré globalement très positif, du point de vue de l'intérêt montré envers ce type de données, mais aussi des résultats obtenus en termes de progression dans la compréhension orale d'extraits naturels de langue parlée et dans l'acquisition d'une bonne capacité d'analyse des phénomènes interactionnels. Lors des entretiens oraux effectués au moment de l'examen, la majorité des étudiants ont pu comprendre et analyser aisément plusieurs phénomènes de la langue parlée à partir d'extraits d'interactions naturelles qu'ils n'avaient jamais entendus auparavant, ce qui n'aurait jamais été possible sans un travail de sensibilisation et d'entraînement préalable.

93 En réponse aux demandes de certains enseignants, nous avons ajouté un onglet "Actions langagières » qui présente un découpage de chacun des extraits en séquences interactionnelles et en actions pour bien faire comprendre leur déroulement et constituer ensuite des collections de certains procédés identifiés par les enseignants comme problématiques pour les apprenants. Nous souhaitons expliciter également les marqueurs de l'oral et leurs fonctions, le rôle des chevauchements, les marques d'adresse, les reformulations ou les hétéro-répétitions dans chacun des quarante extraits pour aider l'enseignant à appréhender les différentes composantes d'une 
interaction. Dans une approche complémentaire, nous développons actuellement, en concertation avec notre réseau partenaire et les associations engagées dans les enjeux d'intégration, une application CORAIL destinée à l'apprenant pour l'aider à comprendre en contexte les interactions sociales du quotidien qu'elles soient privées ou professionnelles; cet outil sera disponible sur téléphone portable, le support aujourd'hui le plus répandu en France et à l'étranger.

\section{BIBLIOGRAPHIE}

ALBERDI, C., ETIENNE, C., JOUIN-CHARDON, É., 2018, « Les apports des corpus d'interactions naturelles en situation de classe : enjeux et pratiques », Action Didactique 1, p. 55-70.

ANDRÉ, V., 2016, « FLEURON : Français Langue Étrangère Universitaire - Ressources et Outils Numériques. Origine, démarches et perspectives », Mélanges Crapel 37, p. 69-92.

ANDRÉ, V., 2006, Construction collaborative du discours au sein de réunions de travail en entreprise : de l'analyse micro-linguistique à l'analyse socio-interactionnelle. Le cas d'un parc départemental de l'équipement, Volume I, Thèse de Doctorat en Sciences du Langage, Université Nancy 2, École Doctorale « Langage, temps, Société ».

ASTON, G. (eds.), 2001, Learning with Corpora, Bologna : Cooperativa Libraria Universitaria Editrice ; Houston TX : Athelstan.

Boulton, A. (eds), 2009, Des Documents Authentiques Oraux aux Corpus : Questions d'Apprentissage en Didactique des Langues, Mélanges CRAPEL 31.

BOUlTON, A., TYNE, H., 2014, Des documents authentiques aux corpus : démarches pour l'apprentissage des langues, Paris : Didier.

BOUlTON, A., TYNE, H., 2013, « Corpus linguistics and data-driven learning : a critical overview », Bulletin suisse de linguistique appliquée 97, p. 97-118.

BOUQUET, S., BEACCO, J.-C., PORQUIER, R., 2004, Les référentiels - Niveau B2, Paris : Didier.

CASSIN, B., 2014, « Fictions de l'origine (entretien réalisé par Quinou) », Vacarme 67, p. 221-248.

Conseil de l'Europe, 2018, Cadre européen commun de référence pour les langues : apprendre, enseigner, évaluer, Volume complémentaire avec de nouveaux descripteurs, Paris : Didier.

DEBAISIEUX, J.-M., 2009, « Des documents authentiques oraux aux corpus : un défi pour la didactique du FLE », Mélanges CRAPEL 31, p. 35-56.

ÉTIENNE, C., JOUIN, É., 2019, « Constituer des ressources pédagogiques pour enseigner le français oral à partir des recherches menées en interaction », dans L. Gajo, J.-M. Luscher, I. Racine, F. Zay (éds.), Variation, plurilinguisme et évaluation en FLE, Berne : Peter Lang, Collection Sprache, Mehrsprachigkeit und sozialer Wandel, p. 225-240.

GOFFMAN, E., 1973, La mise en scène de la vie quotidienne. La présentation de soi, Paris : Minuit.

HOLEC, H., 1990, « Des documents authentiques, pour quoi faire ? », Mélanges pédagogiques, p. 65-76. 
ICOR GROUPE (ÉTIENNE, C., BRUXELLES, S., JOUIN-CHARDON, É., MONDADA, L.), 2018, « Les hétéro-répétitions dans une interaction orale : définition et conception d'un outil de détection automatique ", dans É. Richard (éd.), Des organisations dynamiques de l'oral, Berne : Peter Lang.

ICOR GROUPE, GUINAMARD, I., 2015, « "Du coup” dans l'interaction orale en français : description des usages situés à partir d'une base de données multimédia, et considérations didactiques ", dans I. Guinamard, É. Jouin-Chardon, M. Rispail, V. Traverso, T. D. Thai (eds), Langues parlées, interactions sociales. Une variété d'usages pour l'apprentissage du français, Paris : L'Harmattan, p. 131-153.

GARFINKEL, H., 1984, Studies in Ethnomethodology, Polity Press : Oxford.

GIROUD, A., SURCOUF, C., 2016, « De "Pierre, combien de membres avez-vous ? ” à "Nous nous appelons Marc et Christian" : réflexions autour de l'authenticité dans les documents oraux des manuels de FLE pour débutants », Congrès Mondial de Linguistique Française, p. 1-18.

HORLACHER, A.-S., 2015, La dislocation à droite revisitée. Une approche interactionniste, Bruxelles : De Boeck Supérieur.

JOHNS, T., 1991, "Should you be persuaded: two examples of data-driven learning », dans T. Johns, P. King (eds.), Classroom Concordancing. English Language Research Journal 4, p. 1-16.

KERBRAT-ORECCHIONI, C., TRAVERSO, V., 2009, Les interactions en site commercial. Invariants et variations, Paris : ENS Éditions.

KERBRAT-ORECCHIONI, C., TRAVERSO, V., 2004, « Types d'interaction et genres de l'oral », Langages 153, 41-51.

KRASHEN, S. D., TERRELL, T. D., 1983, The natural approach: Language acquisition in the classroom, New York : Pergamon Press.

LHOTE, É., 1987, À la découverte des paysages sonores des langues, Paris : Les Belles Lettres.

LHOTE, É., 1995, Enseigner l'oral en interaction. Percevoir, écouter, comprendre, Paris : Hachette.

LAURET, B., 2007, Enseigner la prononciation du français : questions et outils, Paris : Hachette.

MONDADA, L., 2017, « Le défi de la multimodalité en interaction », Revue française de linguistique appliquée XXII/2, p. 71-87.

MONDADA, L., 2014, « The local constitution of multimodal resources for social interaction », Journal of Pragmatics 65, p. 137-156.

PEKAREK DOEHLER, S., 2005, « De la nature située des compétences en langue », dans J.-P. BRONCKART et al. (eds.), Repenser l'enseignement des langues : comment identifier et exploiter les compétences, Villeneuve d'Ascq : Presses Universitaires du Septentrion, p. 41-68.

PEKAREK DOEHLER, S., 2006, « Compétence et langage en action », Bulletin Suisse de Linguistique Appliquée 84, p. 9-45.

RAVAZZOLO, E., TRAVERSO, V., JOUIN, É., VIGNER, G., 2015, Interactions, dialogues, conversations : l'oral en français langue étrangère, Paris : Hachette, coll. F.

RIEGEL, M., PELLAT, J.-C., RIOUL, R., 1994, Grammaire méthodique du français, Paris : PUF.

SACKS, H., SCHEGLOFF, E. A., JEFFERSON, G., 1974, « A simplest systematics for the organization of turntaking for conversation », Language 50/4, p. 696-735. 
THOMAS, A., GRANFELDT, J., JOUIN-CHARDON, É., ETIENNE, C., 2016, « Conversations authentiques et CECR : compréhension globale d'interactions naturelles par des apprenants de FLE », Cahiers de l'AFLS 20/2, p. 1-44.

TICCA, A. C., TRAVERSO, V. (eds.), 2015, « Traduire et interpréter en situations sociales. Santé, éducation, justice », Langage \& société 153, Paris : Maison des Sciences de l'Homme.

TRAVERSO, V., 1998, La conversation familière, Lyon : Presses universitaires de Lyon.

TRAVERSO, V., 2011, « Chevauchements de parole, résolution et réparation dans la conversation en ligne », dans C. Develotte, R. Kern, M.-N. Lamy (éds.), Décrire la conversation en ligne, Le face à face distanciel, Lyon : ENS Editions, p. 117-145.

TRAVERSO, V., 2016, Décrire le français parlé en interaction, Paris : Ophrys.

TRAVERSO, V., TICCA, A. C., URSI, B., 2018, «Invitations in French : A complex and apparently delicate action ", Journal of Pragmatics 125, p. 164-179.

\section{NOTES}

1. Interactions Corpus Apprentissages Représentations, CNRS, ENS de Lyon, Université Lyon 2, http ://icar.cnrs.fr.

2. CLAPI Corpus de LAngue Parlée en Interaction, http ://clapi.icar.cnrs.fr.

3. CLAPI-FLE Corpus de LAngue Parlée en Interaction pour le FLE, http ://clapi.icar.cnrs.fr/FLE.

4. D'après le même test de langue Dialang, https ://dialangweb.lancaster.ac.uk/.

5. Le projet PFC-EF , https ://www.projet-pfc.net/le-projet-pfc-ef.

6. Par «multimodalité » nous entendons « la capacité des participants à utiliser des ressources langagières, sonores, visuelles et corporelles pour communiquer» (Ravazzolo et al. 2015 : 36).

7. http://clapi.icar.cnrs.fr/FLE/liste_extraits.php

\section{RÉSUMÉS}

Pour faciliter l'enseignement du français oral en situation de classe, la plateforme CLAPI-FLE rend disponible une quarantaine d'extraits vidéo et audio de corpus naturels d'interactions correspondant à des situations du quotidien dans des contextes privés ou professionnels variés : repas entre amis ou en famille, réunions professionnelles, consultations médicales, achats dans des commerces, visites guidées, sessions de jeux, invitations et appels téléphoniques. En parallèle de ces extraits décrits, transcrits et didactisés, les interactionnistes du laboratoire ICAR construisent, en collaboration avec un réseau de partenaires didacticiens, de nouvelles ressources thématiques basées sur leurs travaux de recherche et répondant aux besoins des enseignants (Ravazzolo et al. 2015, Thomas et al. 2016, Alberdi et al. 2018). Cet article développera l'approche méthodologique qui a conduit à cette plateforme et analysera l'exploitation de ces ressources et leur articulation en situation de classe pour des objectifs d'enseignement variés en français et en linguistique française. Il apportera également un éclairage sur les retours d'expérience vécus par les étudiants. 
To facilitate the teaching of spoken French in class, the CLAPI-FLE platform gives access to some forty video and audio extracts of naturally occurring interactions. They are based on everyday situations and recorded in various private or professional contexts: dinner talks with friends or family, business meetings, medical consultations, purchases in small shops, guided tours, games, invitations and phone calls, etc. In addition to these extracts, which are described, transcribed and presented with pedagogical guidelines, the interactionists of the ICAR laboratory, in collaboration with a network of didactician partners, designed new thematic resources. They are based on their research and aim to match the needs of language teachers (Ravazzolo et al. 2015, Thomas et al. 2016, Alberdi et al. 2018). This article will develop our methodological approach and analyze how these resources can be used in classes according to different teaching purposes for French and French linguistic learnings. It will also shed light on the learners' experience and perspective.

\section{INDEX}

Mots-clés : interaction ; corpus oraux ; Français Langue Etrangère FLE ; enseignement ;

linguistique

Keywords : interaction ; oral corpora ; French as a Foreign Language, FFL ; teaching linguistics

\section{AUTEURS}

\section{ELISA RAVAZZOLO}

Enseignante-chercheuse à l'université de Trento, Italie

\section{CAROLE ETIENNE}

Ingénieure de recherches, Laboratoire ICAR, Lyon 